\title{
Profound Bradycardia With Decreased PEEP
}

\author{
Susan R Wilcox MD, Ankit Kansagra MD, and Jeremy B Richards MD MA
}

\begin{abstract}
An athletic 21-year-old male was admitted to the surgical ICU after sustaining 2 stab wounds to his torso. He had an episode of left lung collapse early in his course, managed with suctioning and increased PEEP, to $15 \mathrm{~cm} \mathrm{H} \mathrm{H}_{2} \mathrm{O}$. He was bradycardic (heart rates 50-60 beats/min) throughout his ICU stay, but when the PEEP was lowered to $5 \mathrm{~cm} \mathrm{H}_{2} \mathrm{O}$ in preparation for extubation, he developed sinus pauses and his heart rate dropped to 20 beats/min. After a thorough evaluation, the drop in his heart rate was determined to be due to increased vagal tone from increased cardiac output with the decreased PEEP. After premedication with glycopyrrolate, he was successfully extubated the following day, while his heart rate remained at his baseline of 50 beats $/ \mathrm{min}$. We review the physiologic mechanisms of bradycardia due to the removal of mechanical ventilation. Key words: bradycardia; autonomic signaling; cardiovascular reflex; vasovagal reflex; mechanical ventilation. [Respir Care 2013;58(11):e138-e143. (c) 2013 Daedalus Enterprises]
\end{abstract}

\section{Introduction}

Bradycardia in mechanically ventilated, critically ill patients may be an incidental finding or it may represent serious pathology. In mechanically ventilated patients, bradycardia is associated with a relatively limited differential diagnosis. After determining whether a patient's bradycardia is a sinus bradycardia or whether it is due to a bradydysrhythmia, clinicians should consider a broad differential diagnosis, including medication effects, conduction system disease, myocardial damage, and metabolic or endocrinologic abnormalities. ${ }^{1}$ Diagnostic evaluations should be guided by the patient's clinical circumstances. In addi-

Dr Wilcox is affiliated with the Department of Anesthesia, Critical Care, and Pain Medicine, Massachusetts General Hospital, Harvard Medical School, Boston, Massachusetts. Dr Kansagra is affiliated with the Department of Medicine, North Shore Medical Center, Salem, Massachusetts. Dr Richards is affiliated with the Division of Pulmonary, Critical Care and Sleep Medicine, Department of Medicine, Beth Israel Deaconess Medical Center, Harvard Medical School, Boston, Massachusetts.

The authors have disclosed no conflicts of interest. The peer review for this paper was managed by Deputy Editor Richard Branson.

Correspondence: Jeremy B Richards MD MA, Division of Pulmonary, Critical Care and Sleep Medicine, Department of Medicine, Beth Israel Deaconess Medical Center, 330 Brookline Avenue, KS-B23, Boston MA 02215. E-mail: jbrichar@bidmc.harvard.edu.

DOI: 10.4187 respcare.02437 tion to these pathologic processes, young athletic patients are predisposed to slower heart rate, due to increased parasympathetic, or vagal, tone. ${ }^{2,3}$ In general, increased vagal tone is a diagnosis of exclusion in critically ill patients.

We present a case of a young, previously healthy athlete with baseline bradycardia while critically ill and on mechanical ventilation. With increased vagal tone due to stimuli such as coughing or gagging on the endotracheal tube, his bradycardia became profound. The most dramatic manifestation of the patient's vagal response occurred with the reduction of his PEEP from $15 \mathrm{~cm} \mathrm{H}_{2} \mathrm{O}$ to $5 \mathrm{~cm} \mathrm{H}_{2} \mathrm{O}$, with the development of sinus pauses. After a thorough investigation, the ICU and cardiology teams attributed his profound bradycardia to high parasympathetic output in response to his increased cardiac output with the drop in PEEP.

This case report demonstrates several principles of cardiopulmonary physiology in mechanically ventilated patients. Specifically, we review physiologic mechanisms for decreased heart rate due to withdrawal of mechanical ventilation.

\section{Case Report}

A 21-year-old healthy male athlete was brought to the emergency department after suffering 2 stab wounds: one to the superior left trapezius, and another to the left flank, in the posterior axillary line over the lower rib cage. In the emergency department his heart rate ranged from 46 to 
64 beats/min and his systolic blood pressure ranged from 127 to $150 \mathrm{~mm} \mathrm{Hg}$ with diastolic pressures of 55 to $76 \mathrm{~mm} \mathrm{Hg}$. He was found to have a diaphragm injury, a splenic laceration, and a gastric injury. He was taken directly to the operating room, where he underwent exploratory laparotomy, with repair of the gastric, diaphragmatic, and splenic lacerations, and left chest tube placement. He was extubated in the operating room immediately after the surgery, but had to be re-intubated within several minutes, due to respiratory failure with paradoxical respiratory efforts and desaturation.

He was then admitted to the surgical ICU with ventilator settings of pressure support $10 \mathrm{~cm} \mathrm{H}_{2} \mathrm{O}$, PEEP of $8 \mathrm{~cm} \mathrm{H}_{2} \mathrm{O}$, and $\mathrm{F}_{\mathrm{IO}_{2}}$ 0.60. In the surgical ICU his heart rate was initially between $90-100$ beats/min after arriving from the operating room, but over the course of the first night his heart rate decreased to 50-60 beats/min, with systolic blood pressure between 140-150 mm Hg. Propofol and hydromorphone infusions were decreased to determine if they were affecting his heart rate, but his heart rate remained approximately 50 beats/min. He was not receiving any nodal-blocking agents such as beta-blockers, calcium channel blockers, or alpha-2 agonists such as clonidine or dexmedetomidine.

His heart rate was consistently $40-50$ beats/min on postoperative day 2 . With coughing or gagging on the endotracheal tube, his heart rate would transiently drop to $10-$ 20 beats/min. Telemetry monitoring and electrocardiogram revealed sinus bradycardia without other arrhythmias or cardiac conduction deficits, and he had no evidence of ischemia or other myocardial injury. Troponin-T was within the normal range. Echocardiogram revealed a structurally normal heart, with an ejection fraction of $54 \%$.

Given the concern about a vagal response, atropine was placed at the bedside, but it was not used, as his mean arterial pressure never fell below $65 \mathrm{~mm} \mathrm{Hg}$, and the profound bradycardia was never sustained for more than a few minutes. Cardiology was consulted and concurred that his bradycardia was due to a heightened vagal reaction in a young, healthy athlete.

On postoperative day 2 he had an episode of desaturation, and his chest $\mathrm{x}$-ray demonstrated collapse of his left lung (Fig. 1). Endotracheal suctioning evacuated copious mucus plugs, and PEEP was increased to $15 \mathrm{~cm} \mathrm{H}_{2} \mathrm{O}$, resulting in improved lung expansion and oxygen saturation (Fig. 2).

On postoperative day 3, mechanical inspiratory and expiratory support was decreased, in anticipation of liberating him from the ventilator. When his ventilator settings were decreased to pressure support $5 \mathrm{~cm} \mathrm{H}_{2} \mathrm{O}$ and PEEP $5 \mathrm{~cm} \mathrm{H}_{2} \mathrm{O}$, he again developed profound bradycardia (1020 beats/min) with long sinus pauses (Fig. 3). The respiratory therapist quickly increased the PEEP to $15 \mathrm{~cm} \mathrm{H}_{2} \mathrm{O}$ with improvement in his heart rate (Fig. 4).

A second attempt at decreasing the PEEP resulted in profound bradycardia (Fig. 5). However, as his blood pres-

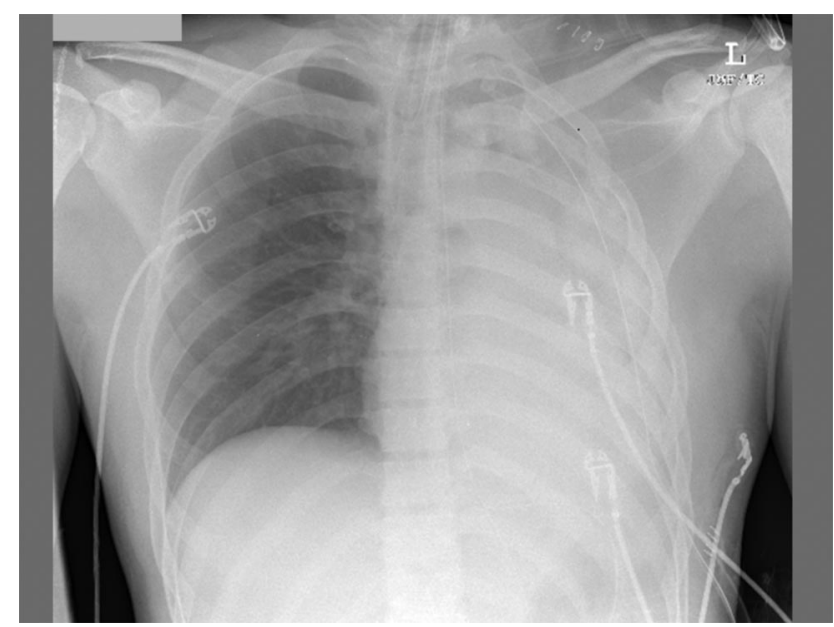

Fig. 1. Anteroposterior portable chest x-ray shows collapse of the left lung due to mucus plugging and derecruitment. The endotracheal tube terminates $\sim 4.5 \mathrm{~cm}$ above the carina.

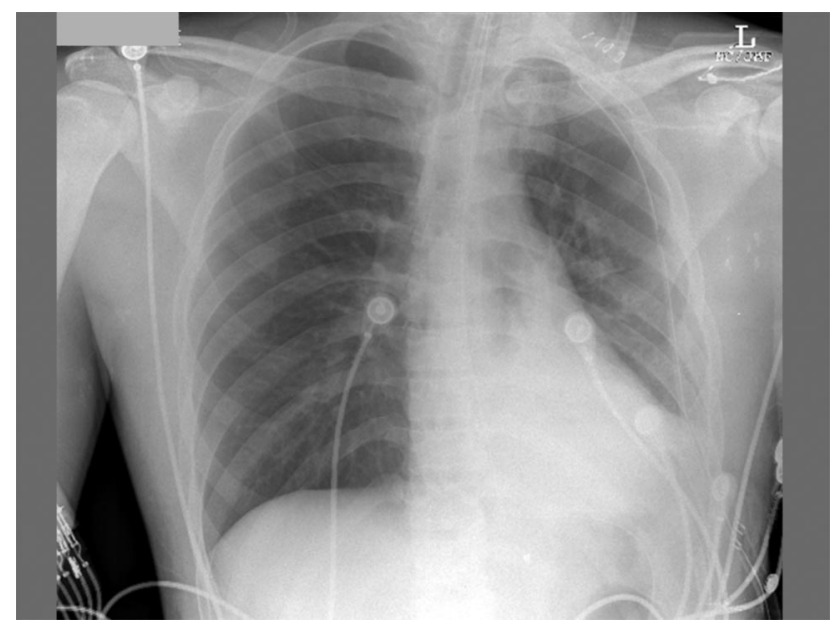

Fig. 2. Portable chest x-ray shows re-expansion of the left lung after suctioning and increased PEEP. The endotracheal tube terminates $\sim 4 \mathrm{~cm}$ above the carina.

sure did not fall, he was monitored on the lower PEEP. His heart rate returned to baseline of 50-60 beats/min after approximately $2 \mathrm{~min}$. The following morning he was premedicated with $0.2 \mathrm{mg}$ of glycopyrrolate to minimize additional vagal responses, and he was successfully extubated without decreasing his heart rate or further pauses. After extubation his heart rate range was $40-70$ beats/min in sinus rhythm, with systolic blood pressure of 120 $150 \mathrm{~mm} \mathrm{Hg}$ and diastolic blood pressure of $50-60 \mathrm{~mm} \mathrm{Hg}$ for the remainder of his hospital course.

\section{Discussion}

Although hemodynamic perturbations frequently occur in mechanically ventilated patients, tachycardia is more 


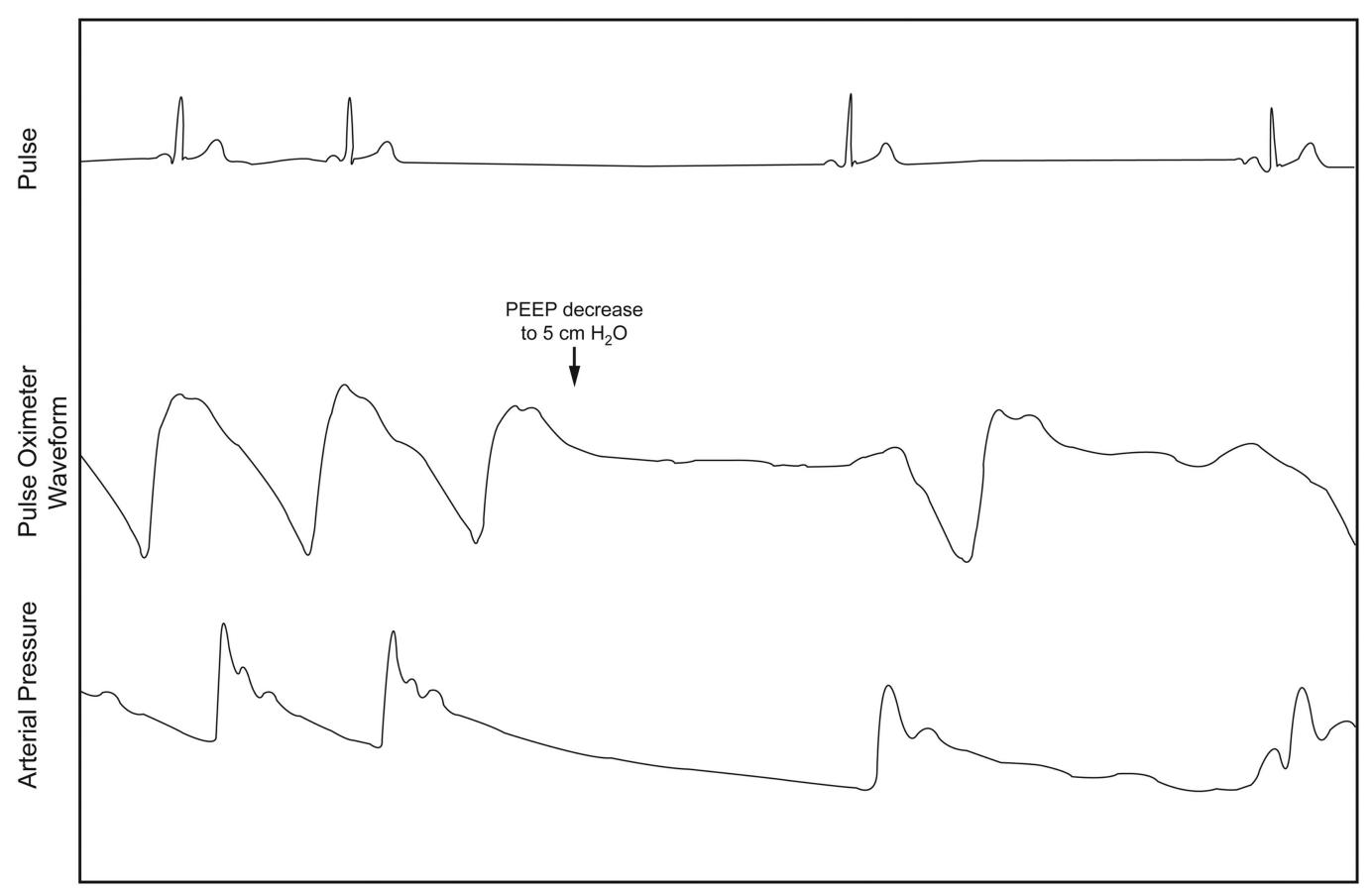

Fig. 3. Telemetry tracing shows the acute onset of sinus pauses and severe bradycardia after decreasing the PEEP to $5 \mathrm{~cm} \mathrm{H}_{2} \mathrm{O}$.

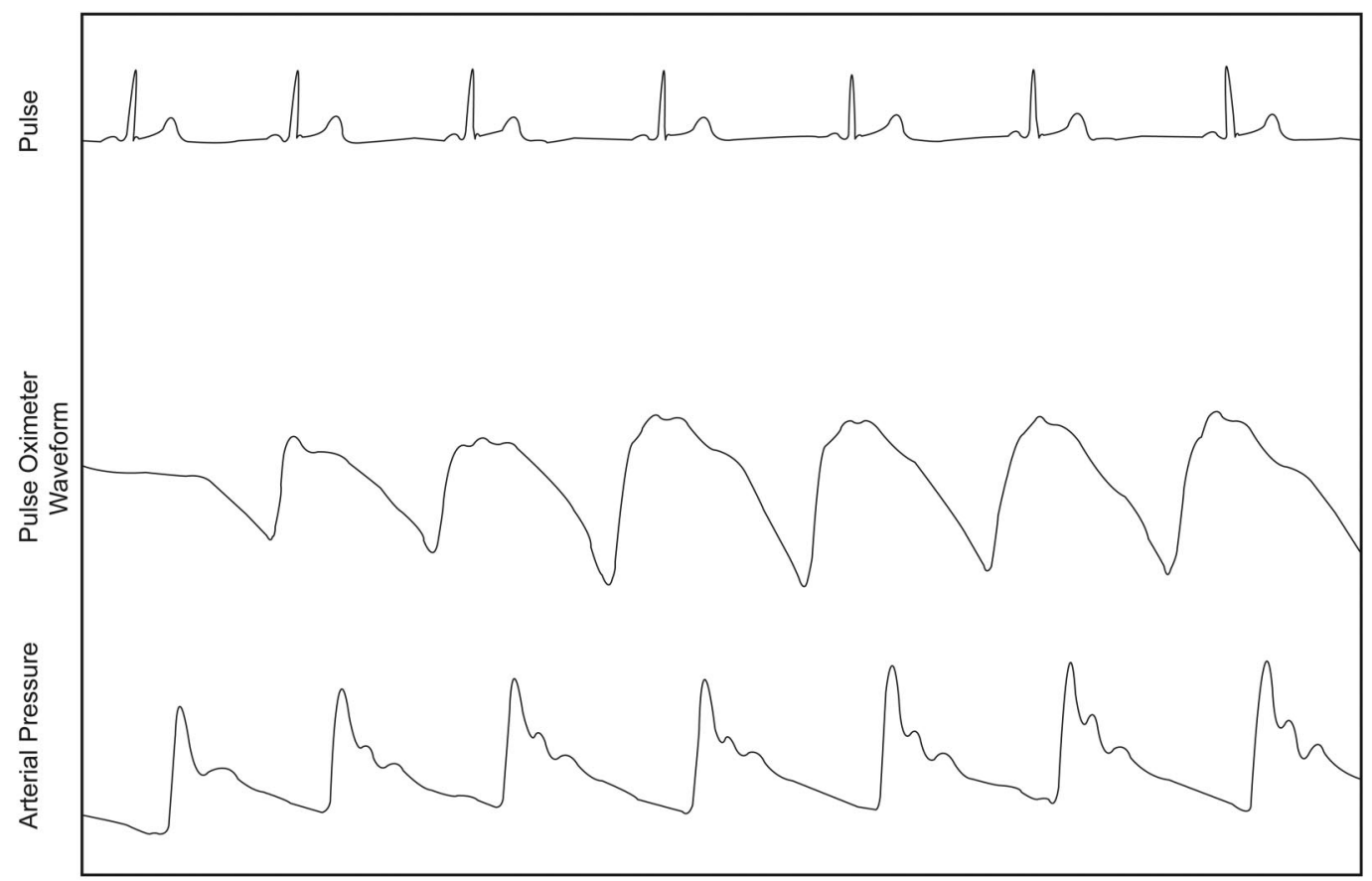

Fig. 4. Telemetry tracing shows improvement in heart rate after increasing the PEEP from 5 to $10 \mathrm{~cm}_{2} \mathrm{O}$.

commonly encountered than bradycardia, and there is a limited differential diagnosis associated with profound bradycardia in intubated patients. One of the most common etiologies of bradycardia in mechanically ventilated pa- tients is the effect of medications, especially nodal blockade or sedatives. In addition to beta-blockers and calcium channel blockers, propofol, dexmedetomidine, and opiates have all been reported to cause bradycardia in mechani- 


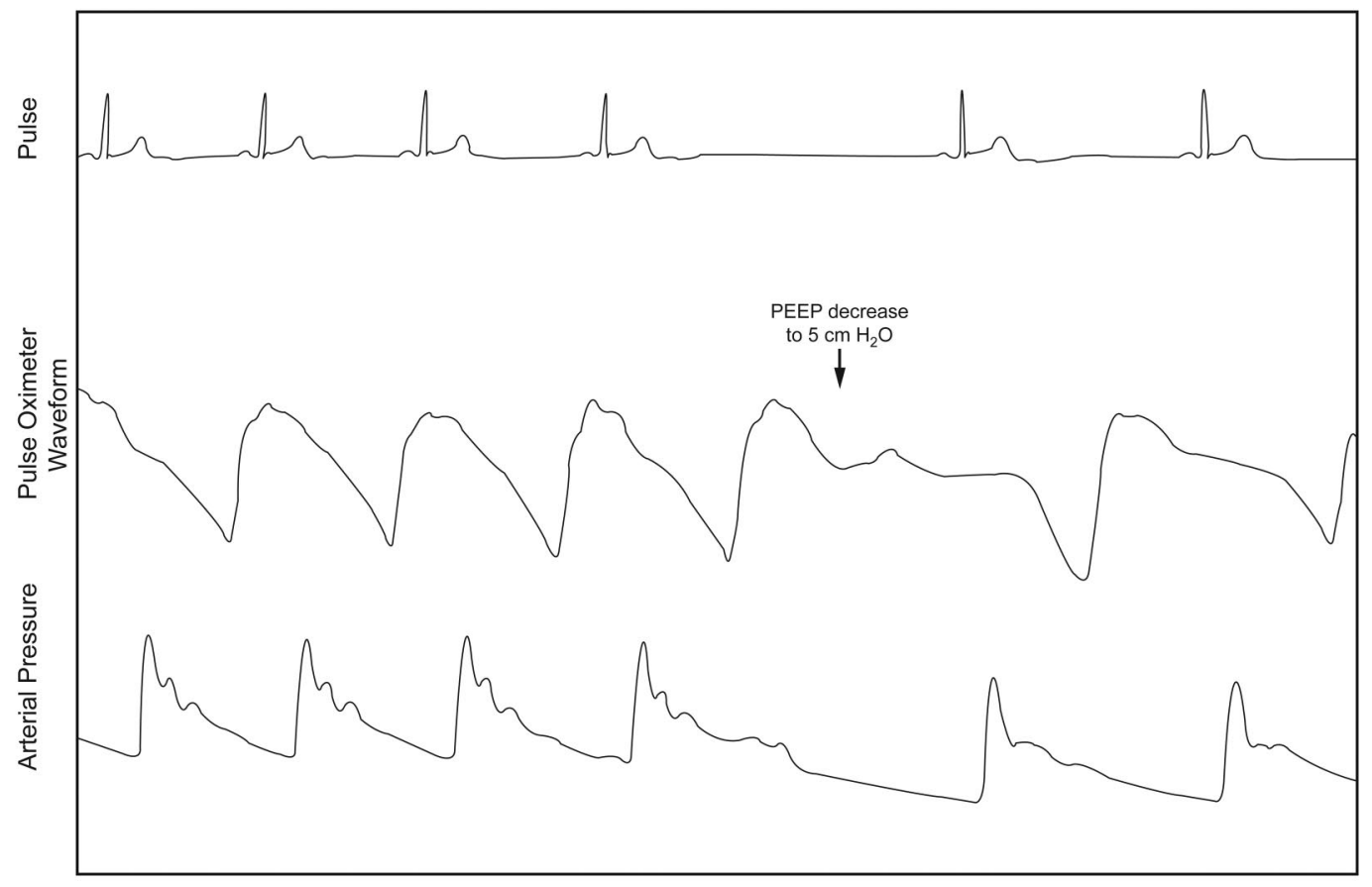

Fig. 5. Telemetry tracing shows recurrent bradycardia after again reducing the PEEP to $5 \mathrm{~cm} \mathrm{H}_{2} \mathrm{O}$.

cally ventilated patients. ${ }^{4}$ Other potential etiologies of bradycardia include electrolyte abnormalities, cardiac conduction disease, spinal cord injury, hypothyroidism, infiltrative cardiac disease, and collagen vascular diseases. ${ }^{1,4,5}$

A diagnostic evaluation for the mechanism of bradycardia targeting potential etiologies should be initiated, starting with a review of the patient's history and physical exam. Clinicians first must differentiate sinus bradycardia from bradydysrhythmias, as this distinction may guide further work-up. Evaluation for cardiac injuries should include serial electrocardiograms and telemetry monitoring, an echocardiogram, cardiac enzyme monitoring, consultation with cardiology, and electrophysiology testing, as indicated.

In addition to these processes, increased vagal tone can result in bradycardia. Given the acuity of critically ill patients, increased vagal tone should be considered a diagnosis of exclusion once other more dangerous etiologies have been ruled out.

\section{Cardiovascular Vasovagal Reflexes}

A primary mechanism for vasovagal bradycardia due to decreased mechanical ventilation pressure is an increase in cardiac output. Mechanical ventilation, and specifically PEEP, decreases intrathoracic blood volume and venous return to the right heart. ${ }^{6,7}$ PEEP can also increase right ventricular afterload and decrease right ventricular output.
In normal, healthy individuals, stroke volume is preload dependent, and therefore increased PEEP can decrease cardiac output by decreasing stroke volume. ${ }^{8}$ The decrease in preload due to PEEP is due to decreased systemic venous return to the right heart and decreased passive right ventricular filling during diastole. ${ }^{3}$ Heart rate increases modestly in response to mechanical ventilation and increased PEEP, to maintain cardiac output. ${ }^{9}$

Conversely, decreasing PEEP will decrease right atrial pressure and increase right ventricular venous return. These changes result in an increase in stroke volume and an increase in cardiac output when transitioning from mechanical ventilation to spontaneous ventilation. ${ }^{10}$ Descriptive physiologic studies have demonstrated that decreased right atrial pressure, as would occur with discontinuation of PEEP, increases right ventricular filling proportional to the pressure withdrawn and to the circulating blood volume. ${ }^{11-13}$ Decreasing PEEP also increases left ventricular afterload. ${ }^{14}$ Therefore, discontinuation of mechanical ventilation and PEEP can decrease cardiac output in patients with impaired myocardial function. ${ }^{15-17}$ However, in a young healthy athlete with a normal ejection fraction and preserved cardiac contractility, the increase in venous return, stroke volume, and cardiac output will be greater than the decrement in cardiac output due to increased left ventricular afterload.

A patient's intravascular volume status contributes to the response to adding or withdrawing PEEP. Intravascular volume depletion would be expected to increase the 


\section{Profound Bradycardia With Decreased PEEP}

tachycardic response to PEEP, and, similarly, the bradycardic response to withdrawing PEEP, as preload is more susceptible to changes in intrathoracic pressure. ${ }^{13}$ The withdrawal of PEEP may result in rapid changes to heart rate, given the immediacy of intrathoracic pressure changes, as compared to other provocative maneuvers such as a volume challenge or a passive straight leg raise, which may take several minutes to affect preload.

There is, however, a brief temporal delay between decreasing or withdrawing PEEP, an increase in right ventricular venous return and cardiac output, and peripheral baroreceptor signaling. Typically, a delay of $8-10$ seconds has been used in experimental models, to allow for equilibration of right and left heart cardiac output. ${ }^{11}$

\section{Pulmonary Stretch Receptors}

With moderate inflation of the lungs from functional residual capacity, the heart rate increases, likely due to increased signaling of slowly adapting pulmonary stretch receptors, resulting in centrally mediated decrease in vagal signaling. ${ }^{18}$ With inflation of the lungs to large volumes, however, reflex bradycardia occurs, possibly due to hyperinflation activating pulmonary $\mathrm{C}$ and $\mathrm{J}$ receptors, which increase vagal signaling. ${ }^{19}$ These heart rate changes are a component of the constellation of systemic physiologic changes caused by the Hering-Breuer inflation reflex, which include decreased inspiratory effort in response in lung inflation. ${ }^{18}$

The Hering-Breuer deflation reflex may be mediated by decreased pulmonary stretch receptor signaling, and is characterized by increased inspiratory effort and breathing frequency, as well as bradycardia. ${ }^{18}$ The Hering-Breuer deflation reflex occurs with large expiratory volumes, and may be considered an extreme manifestation of respiratory sinus arrhythmia, which typically describes modest bradycardia with normal tidal volume exhalation to functional residual capacity. ${ }^{20}$

The importance of pulmonary stretch receptor signaling on cardiovascular and respiratory function in humans is debated, but is generally considered to be relatively minimal at near-normal lung volumes. ${ }^{21}$ In our patient the Hering-Breuer inflation reflex was clearly not playing a role, as he developed bradycardia with decreased PEEP and resultant decreased intrathoracic pressure and tidal volume. While the Hering-Breuer deflation reflex or respiratory sinus arrhythmia may have been contributing to his decreased heart rate with decreased PEEP, these changes in pulmonary stretch receptor signaling were likely occurring in concert with changes in preload, cardiac output, and peripheral baroreceptor signaling associated with the decreased PEEP. ${ }^{18,20}$

\section{Autonomic Nervous Signaling}

The bradycardic response to withdrawal of mechanical ventilation is mediated by increased autonomic nervous system signaling, in response to increased atrial and ventricular stretch. ${ }^{8,22}$ In addition, stimulation of peripheral baroreceptors due to increased left ventricular stroke volume and cardiac output results in a rapid decrease in heart rate, mediated solely by increased efferent vagal output. ${ }^{23,24}$ Vagal stimulation causes local release of acetylcholine, which acts at the sinus node, resulting in a slowed rate of impulse formation. Acetylcholine also acts at the atrioventricular node to slow conduction velocity and lengthen the refractory period. ${ }^{25}$

These mechanisms are demonstrated in this case, as the abrupt reduction in PEEP increased the stroke volume and cardiac output, which stimulated the carotid sinus, activated a strong vagal response, and resulted in acute drop in heart rate. The subsequent increase in heart rate with increasing PEEP demonstrated a clear causal relationship between the PEEP change and the bradycardia.

Few studies have described heart rate variability with withdrawal of mechanical ventilation, and these studies demonstrate heterogeneous associations between weaning ventilator support and changes in heart rate..$^{26-29}$ The data supporting a diagnosis of increased vagal tone and increased baroreceptor receptor signaling leading to bradycardia and sinus pauses in this case were clinical and contextual, as his age and athletic condition before hospitalization support this diagnosis. Electrocardiogram and telemetry monitoring demonstrated sinus rhythm, with consistent $\mathrm{P}$ waves and PR intervals. His heart rate was also lower at night and with any activity that increased parasympathetic tone, such as coughing or gagging on the endotracheal tube. Bradycardia with coughing and gagging likely occurred due to hypopharyngeal and tracheal stimulation rather than due to changes in preload, cardiac output, and peripheral baroreceptor signaling. However, that he developed bradycardia with coughing and gagging demonstrates a high resting vagal tone, and indicates an increased propensity for bradycardia with decreased PEEP.

These clinical observations demonstrate a consistent mechanism for the acute bradycardia in our patient: increased resting vagal tone led to acute bradycardia with PEEP decrease, due to the acute increased right-side venous return, increased atrial and ventricular stretch, and increased stroke volume and cardiac output. In addition, decreased pulmonary stretch receptor signaling and accentuation of vagal signaling and a respiratory sinus arrhythmia may have further contributed to his acute bradycardia with withdrawal of PEEP.

Understanding the mechanisms of bradycardia in this case was essential for safe extubation. The ICU team was aware of the vagal response and premedicated the patient 


\section{Profound Bradycardia With Decreased PEEP}

with glycopyrrolate, and he was extubated with minimal change in heart rate and acceptable blood pressure.

\section{REFERENCES}

1. Kaushik V, Leon AR, Forrester JS Jr, Trohman RG. Bradyarrhythmias, temporary and permanent pacing. Crit Care Med 2000;28(10 Suppl):N121-N128.

2. Smith ML, Hudson DL, Graitzer HM, Raven PB. Exercise training bradycardia: the role of autonomic balance. Med Sci Sports Exerc 1989;21(1):40-44

3. Iellamo F, Legramante JM, Pigozzi F, Spataro A, Norbiato G, Lucini D, Pagani M. Conversion from vagal to sympathetic predominance with strenuous training in high-performance world class athletes. Circulation 2002;105(23):2719-2724.

4. Devlin JW, Mallow-Corbett S, Riker RR. Adverse drug events associated with the use of analgesics, sedatives, and antipsychotics in the intensive care unit. Crit Care Med 2010;38(6 Suppl):S231-S243.

5. Trappe HJ, Brandts B, Weismueller P. Arrythmias in the intensive care patient. Curr Opin Crit Care 2003;9(5):345-355.

6. Pinsky MR. Cardiovascular issues in respiratory care. Chest 2005; 128(5 Suppl 2):592S-597S

7. Beach T, Millen E, Grenvik A. Hemodynamic response to discontinuance of mechanical ventilation. Crit Care Med 1973;1(2):85-90.

8. Pinsky MR. Instantaneous venous return curves in an intact canine preparation. J Appl Physiol 1984;56(3):765-771.

9. Blevins SS, Connolly MJ, Carlson DE. Baroreceptor-mediated compensation for hemodynamic effects of positive end-expiratory pressure. J Appl Physiol 1999;86(1):285-293.

10. Frazier SK, Moser DK, Stone KS. Heart rate variability and hemodynamic alterations in canines with normal cardiac function during exposure to pressure support, continuous positive airway pressure, and a combination of pressure support and continuous positive airway pressure. Biol Res Nurs 2001;2(3):167-174.

11. Guyton AC, Lindsey AW, Abernathy B, Richardson T. Venous return at various right atrial pressures and the normal venous return curve. Am J Physiol 1957;189(3):609-615.

12. Viquerat CE, Righetti A, Suter PM. Biventricular volumes and function in patients with adult respiratory distress syndrome ventilated with PEEP. Chest 1983;83(3):509-514.

13. Tyberg JV, Taichman GC, Smith ER, Douglas NW, Smiseth OA, Keon WJ. The relationship between pericardial pressure and right atrial pressure: an intraoperative study. Circulation 1986;73(3):428432.

14. Pinsky MR, Desmet JM, Vincent JL. Effect of positive end-expiratory pressure on right ventricular function in humans. Am Rev Respir Dis 1992;146(3):681-687.
15. Buda AJ, Pinsky MR, Ingels NB, Daughters GT, Stinson EB, Alderman EL. Effect of intrathoracic pressure on left ventricular performance. N Engl J Med 1979;301(9):453-459.

16. Schuster S, Erbel R, Weilemann LS, Lu WY, Henkel B, Wellek S, et al. Hemodynamics during PEEP ventilation in patients with severe left ventricular failure studied by transesophageal echocardiography. Chest 1990;97(5):1181-1189.

17. Fallahi JL, Valtier B, Beauchet A, Bourdarias JP, Jardin F. Does positive end-expiratory pressure ventilation improve left ventricular function? A comparative study by transesophageal echocardiography in cardiac and noncardiac patients. Chest 1998;114(2):556-562.

18. Shepherd JT. The lungs as receptor sites for cardiovascular regulation. Circulation 1981;63(1):1-10.

19. Strong EB, Green JF. Multireceptor activation of the pulmonary chemoreflex. J Appl Physiol 1991;70(1):368-370.

20. Larsen PD, Tzeng YC, Sin PY, Galletly DC. Respiratory sinus arrhythmia in conscious humans during spontaneous respiration. Respir Physiol Neurobiol 2010;174(1-2):111-118.

21. Binks AP, Paydarfar D, Schachter SC, Guz A, Banzett RB. High strength stimulation of the vagus nerve in awake humans: a lack of cardiorespiratory effects. Respir Physiol 2001;127(2-3):125-133.

22. Bernardi L, Keller F, Sanders M, Reddy PS, Griffith B, Meno F, Pinsky MR. Respiratory sinus arrhythmia in the denervated human heart. J Appl Physiol 1989;67(4):1447-1455.

23. Grimm DR. Neurally mediated syncope: a review of cardiac and arterial receptors. J Clin Neurophysiol 1997;14(3):170-182.

24. Paintal AS. Vagal sensory receptors and their reflex effects. Physiol Rev 1973;53(1):159-227.

25. Imaizumi S, Mazgalev T, Dreifus LS, Michelson EL, Miyagawa A, Bharati S, Lev M. Morphological and electrophysiological correlates of atrioventricular nodal response to increased vagal activity. Circulation 1990;82(3):951-964

26. Shen HN, Lin LY, Chen KY, Kuo PH, Yu CJ, Wu HD, Yang PC. Changes of heart rate variability during ventilator weaning. Chest 2003;123(4):1222-1228

27. Valipour A, Schneider F, Kössler W, Saliba S, Burghuber OC. Heart rate variability and spontaneous baroreflex sequences in supine healthy volunteers subjected to nasal positive airway pressure. J Appl Physiol 2005;99(6):2137-2143.

28. Papaioannou VE, Chouvarda I, Maglaveras N, Dragoumanis C, Pneumatikos I. Changes of heart and respiratory rate dynamics during weaning from mechanical ventilation: a study of physiologic complexity in critically ill patients. J Crit Care 2011;26(3):262-272.

29. Beda A, Carvalho NC, Guldner A, Koch T, de Abreu MG. Mechanical ventilation during anaesthesia: challenges and opportunities for investigating the respiration-related cardiovascular oscillations. Biomed Tech (Berl) 2011;56(4):195-206. 